\title{
Models of Disk Evolution: Confrontation with Observations
}

\author{
Rychard Bouwens \\ Physics Department, University of California, Berkeley, CA 94720; \\ bouwens@astro.berkeley.edu \\ $\&$ \\ Joseph Silk \\ Astrophysics, Department of Physics, University of Oxford, Oxford OX1 3RH UK and \\ Astronomy and Physics Departments, and Center for Particle Astrophysics, University of \\ California, Berkeley, CA 94720; silk@astro.berkeley.edu
}

\begin{abstract}
We present simple models for disk evolution based on two different approaches: a forward approach based on predictions generic to hierarchical models for structure formation (e.g., Mo, Mao, \& White 1998) and a backwards approach based on detailed modeling of the Milky Way galaxy (e.g., Bouwens, Cayón, \& Silk 1997). We normalize these models to local observations and predict high-redshift luminosities, sizes, circular velocities, and surface brightnesses. Both approaches yield somewhat similar predictions for size, surface brightness, and luminosity evolution though they clearly differ in the amount of number evolution. These predictions seem to be broadly consistent with the high-redshift observations of Simard et al. (1999), suggesting that the $B$-band surface brightness of disks has indeed evolved by $\sim 1.5^{m}$ from $z \sim 0$ to $z \sim 1$ similar to the models and is not an artifact of selection effects as previously claimed. We also find a lack of low surface brightness galaxies in several high redshift samples relative to model predictions based on local samples (de Jong \& van der Kruit 1994; Mathewson, Ford, \& Buchhorn 1992).
\end{abstract}

Subject headings: galaxies: evolution

\section{Introduction}

Over the last several years, there has been a steady increase in the number and quality of observations available for disk galaxies from $z=0$ and $z=1$. Schade et al. $(1995,1996)$, 
using early ground and space based images of galaxies from the Canada-France Redshift Survey (CFRS), found a net increase in the surface brightness of galaxies to $z \sim 1$. Along the same lines, Roche et al. (1998), compiling 347 galaxies from the Medium Deep Survey and other surveys, concluded that disk galaxies had undergone a net evolution in surface brightness and a net devolution in size. Lilly et al. (1998), using structural parameters extracted from HST images of the combined CFRS and LDSS2 sample, concluded that there has been essentially no evolution in large disks out to $z \sim 1$. As a preliminary effort as part of the DEEP survey, Vogt et al. $(1996,1997)$ found little evolution in the Tully-Fisher relationship $\left(<0.3^{m}\right)$ out to $z \sim 1$. More recently, these observations have been augmented by the DEEP sample with 197 galaxies from the Groth strip to $I<23.5,1.5$ magnitudes deeper than the LDSS2-CFRS sample. In a first paper, Simard et al. (1998) concluded that there had been little evolution in the disk surface brightness distribution to $z \sim 1$ contrary to previous claims.

A number of different approaches have been proposed for making specific predictions about disk evolution. Mo, Mao, \& White (1998a) showed how the standard paradigm for hierarchical growth of structure combined with simple assumptions about angular momentum conservation led to simple scaling relationships for the change in disk properties as a function of redshift. Other authors (Ferrini et al. 1994; Prantzos \& Aubert 1995; Prantzos \& Silk 1998; Boissier \& Prantzos 1999; Chiappini, Matteucci \& Gratton 1997), taking more of a backwards approach to the problem, used detailed studies of the profiles of the Milky Way and other nearby galaxies to propose radially dependent models of star formation in disk galaxies, models which could be used to make detailed predictions about high-redshift disk evolution.

Already there have been a number of elegant studies in which both the backwards approach (Cayón, Silk, \& Charlot 1996; Bouwens, Cayón, \& Silk 1997; Roche et al. 1998) and the forwards approach (Mao, Mo, \& White 1998; Steinmetz \& Navarro 1999; Contardo, Steinmetz, \& Fritze-von Alvensleben 1998; van den Bosch 1998; Mo, Mao, \& White 1998b) have been used to interpret the observations available for disk galaxies, mostly to $z \sim 1$.

Unfortunately, none of these studies considered the important effect that a large spread in surface brightness could have on the interpretation of these observations, particularly the potentially large fraction of low surface brightness galaxies. In some studies, the surface brightness selection effects at low and high redshift were simply ignored, and in others, e.g., Roche et al. (1998), the spread was limited to $0.3 \mathrm{mag} / \mathrm{arcsec}^{2}$ about Freeman's law (Freeman 1970). Clearly, given the apparent large numbers of low surface brightness galaxies seen locally, it is quite logical to wonder if these galaxies are detectable in current high redshift surveys. Indeed, one might wonder whether these galaxies or the 
observed correlation between luminosity and surface brightness may have already affected the interpretation of high redshift observations. In light of the recent claim by Simard et al. (1999) that the apparent surface brightness evolution thus far inferred to $z \sim 1$ is completely due to surface brightness selection effects, such a study would seem to be especially timely. Secondly, none of these studies directly compared the predictions of the forward and backward approaches using the same observations. Simple comparisons of the scaling expected in surface brightness, size, luminosity, and number are useful for interpreting the high redshift observations.

To address these shortcomings, we shall therefore consider implementations of both approaches, normalize them to the observed $z \sim 0$ size-luminosity relationship, compare their predictions, and consider how each of them fares at explaining the observed disk evolution out to $z \sim 1$ incorporating all the selection effects as they are best understood. We commence by presenting our models $(\S 2)$ and the observational samples with which we compare $(\S 3)$. We present the results $(\S 4)$, discuss them $(\S 5)$, and then summarize our conclusions $(\S 6)$. Throughout this study, we use $H_{0}=50 \mathrm{~km} / \mathrm{s} / \mathrm{Mpc}$ unless otherwise noted.

\section{Models}

We begin by sketching the base $z=0$ model to be used for both the models which follow. We use a set of gaussian LFs based on those presented in Binggeli, Sandage, and Tammann (1988):

$$
\phi(M) d M=\frac{\phi_{0}}{2 \pi \sigma_{M}} \exp \left(-\left(\frac{M-M_{*}}{\sigma_{M}}\right)^{2}\right) d M
$$

We adjusted the bulge-to-total $(B / T)$ distributions of these galaxy types to obtain fair agreement with the de Jong \& van der Kruit (1994) sample. Finally, we adjusted the luminosity function so there was rough agreement with the combined Sabc and Sdm luminosity functions presented in Pozzetti, Bruzual, \& Zamorani (1996). We present our parameterized populations in Table 1.

For the above luminosity functions, we convert $z=0 B$-band luminosity to mass using a constant mass-to-light ratio, where the mass of a $M_{b_{J}}=-21.1$ galaxy is $1.1 \cdot 10^{12} M_{\odot}$. We assume a log-normal scatter of 0.3 dex to reproduce the observed Tully-Fisher scatter though variation in the formation times (van den Bosch 1998) and concentration indexes (Avila-Reese, Firmani, \& Hernandez 1998) certainly play a role.

To translate this mass into a circular velocity and size, we calculate the time at which the ambient halo formed. Since halos are always accreting more mass and merging with larger halos, there is some ambiguity in defining this, so for simplicity we take it to equal 
the redshift at which half of the mass in a halo has been assembled. We determine the distribution of formation times using the procedure outlined in Section 2.5.2 of Lacey \& Cole (1993). We take the circular velocity of the halo to be that corresponding to the halo at its formation time using Eq. (14).

Then, given the mass, circular velocity, and luminosity of the $z=0$ disk, we randomly draw the sizes $r_{e}$ from the following distribution:

$$
\phi\left(r_{e}\right) d \log r_{e}=\frac{1}{\sigma_{\lambda} \sqrt{2 \pi}} \exp \left(-\frac{1}{2}\left[\frac{\log r_{e} / r_{e}^{*}-0.4\left(M-M_{*, s}\right)(1 / 3)}{\sigma_{\lambda} / \ln (10)}\right]^{2}\right) d \log r_{e}
$$

where $r_{e}^{*}=6.9 \mathrm{kpc}, \sigma_{\lambda}=0.37$, and $M_{*, s}=-21.1$. We provide a basic observational and theoretical motivation for this scaling in $\S 2.1$. Note that here the surface brightness is proportional to $L^{1 / 3}$, that the spread in the size distribution is proportional to the spread in the distribution of $\lambda$, and that the scale length (surface brightness) of the average $L_{*}$ galaxy is exactly equal to that predicted by Freeman's law.

We assume that the SED of disks and bulges is identical to that of a 10 Gyr-old stellar population with an e-folding time of 4.5 Gyr and a $\tau_{B}=0.3$ foreground dust screen, the extinction curve being that of Calzetti (1997). For simplicity, we assume this SED is constant independent of time. We use the Bruzual \& Charlot tables from the Leitherer et al. (1996) compilation for this calculation. In the following models, we evolve the size, number, and luminosity of all galaxy types using simple single-valued functions of redshift:

$$
\begin{gathered}
R(z)=R(0) E_{R}(z) \\
N(z)=N(0) E_{N}(z) \\
L(z)=L(0) E_{L}(z)
\end{gathered}
$$

Table 1. Model parameters for disk population.

\begin{tabular}{ccccc}
\hline \hline$\phi_{o}$ & $\sigma_{M}$ & $M_{b_{j}}^{*}$ & $B / T$ & $\sigma_{B / T}$ \\
\hline 2.0 & 1.1 & -19.4 & 0.25 & 1.8 \\
2.5 & 1.1 & -19.6 & 0.08 & 0.5 \\
8.0 & 1.3 & -18.4 & 0.04 & 0.25 \\
24.0 & 1.3 & -16.0 & 0.01 & 0.25 \\
\hline
\end{tabular}


For simplicity, we assume similar scalings in the properties of bulges as a function of time.

Since the color and luminosity of disks has been shown to be correlated with inclination, it is reasonable to suppose that disks are not transparent. Unfortunately, there is much controversy concerning the degree to which disks are or are not transparent. For better or worse, we will side-step this controversy and simply adopt the Tully \& Fouqué (1985) prescription for extinction in the $B$ band:

$$
A_{B}=-2.5 \log \left(f(1-\exp (-\tau \sec i))+(1-2 f)\left(\frac{1-\exp (-\tau \sec i)}{\tau \sec i}\right)\right)
$$

where $\tau=0.55, f=0.25$, and $i$ is the inclination of the disk, 0 corresponding to a face-on disk. We shall assume our model galaxies are always observed at an inclination of $70 \mathrm{deg}$ and therefore always correct the observations to this inclination for comparison with the models. In the $B$ band, this corresponds to an extinction correction of $0.67^{\mathrm{m}}$.

\subsection{Hierarchical Model (Forwards Approach)}

The use of simple scaling relationships between the properties of disks and the halos in which they live has provided a relatively successful way of explaining both the internal correlations between disk properties and their evolution to high redshift. In this picture developed by Fall \& Estathiou (1980) and revived more recently by Dalcanton et al. (1997) and Mo et al. (1998) among others, the bivariate mass and angular momentum distribution nicely translates into a luminosity and surface brightness relationship for disk galaxies, mass translating directly into luminosity and the dimensionless angular momentum translating directly into surface brightness.

This picture has had much success in explaining the internal correlations between size, circular velocity, and mass. For example, De Jong \& Lacey (1999) recently showed that the observed local bivariate luminosity-size distribution is nicely fit by this picture, albeit with a slightly smaller scatter in surface brightness than might otherwise be expected. For constant mass-to-light ratios, the rough $M \propto V_{c}^{3}$ relationship for halos provides a relatively natural explanation for the luminosity-circular velocity (Tully-Fisher) relationship (Dalcanton, Spergel, \& Summers 1997a; Mo et al. 1998a; Steinmetz \& Navarro 1999; Contardo et al. 1998). Finally, the $R \propto V_{c}$ relationship found in galaxy halos is similarly observed in the disk population (Courteau 1997).

On the other hand, this picture says little, if anything, about how the gas disk evolved over time and therefore what its local properties (i.e., spatial variations in the metallicity, color, stellar ages, gas density, etc.) or global properties (total gas mass) are, 
and therefore comparisons of this sort will depend upon the model adopted, whether it be one of the popular semi-analytic approaches (Cole et al. 1999, Somerville \& Primack 1998) or a full N-body hydrodynamical simulation (e.g., Contardo et al. 1998). Moreover, it is now apparent that the simple scaling model is fundamentally flawed with regard to the implementation of galaxy formation theory as revealed by high resolution numerical simulations (Moore et al. 1999; Navarro \& Steinmetz 1999; Steinmetz \& Navarro 1999). Since it however is the only detailed model available, it is imperative to fully explore comparisons with data, properly incorporating observational selection effects, in order to establish the correct basis for ultimately refining the model.

For a detailed discussion of this picture, i.e., the idea that simple scalings in the properties of halos lead to simple scalings in the properties of disks, the reader is referred to Mo et al. (1998a) and later papers by the same authors. For the sake of clarity, we shall review some of this material. In the standard spherical collapse model for an Einstein-de Sitter universe, the density of the collapsed halo is $18 \pi^{2} \approx 178$ times the critical density of the universe at collapse time (see also Gunn \& Gott 1972; Bertschinger 1985; Cole \& Lacey 1996), but depends on the density of universe through the parameter $x=1-\Omega(z)$. Expressing the result in terms of the super critical density parameter $\Delta_{c}$

$$
\frac{M}{\frac{4}{3} \pi r_{v i r}^{3}}=\Delta_{c} \rho_{c}
$$

Bryan \& Norman (1998) found that for $\Omega+\Omega_{\Lambda}=1$,

$$
\Delta_{c} \approx 18 \pi^{2}+82 x-39 x^{2}
$$

for $\Omega_{\Lambda}=0$,

$$
\Delta_{c} \approx 18 \pi^{2}+60 x-32 x^{2}
$$

where $x=\Omega(z)-1$.

Using the virial theorem, it is possible to write equations to relate the mass, radius, and circular velocity of each halo. As in Somerville \& Primack (1998), it can be shown that

$$
V_{v i r}^{2}=\frac{G M}{r_{v i r}}-\frac{\Omega_{\Lambda}}{3} H(z)^{2} r_{v i r}^{2}
$$

where $r_{v i r}$ is the halo size, $V_{v i r}$ is the circular velocity of the halo at $r_{v i r}, G$ is Newton's constant, and

$$
H\left(z_{f}\right)=H_{0} \sqrt{\Omega_{\Lambda, 0}+\left(1-\Omega_{0}-\Omega_{\Lambda, 0}\right)\left(1+z_{f}\right)^{2}+\Omega_{0}\left(1+z_{f}\right)^{3}} .
$$

Using the fact that $M=\Delta_{c} \rho_{c} \frac{4}{3} \pi r_{v i r}^{3}=\Delta_{c} H(z)^{2} \frac{1}{2 G} r_{v i r}^{3}$, we can rewrite this as

$$
V_{v i r}^{2}=\frac{1}{2}\left(\Delta_{c}-\Omega_{\Lambda}\right) H(z)^{2} r_{v i r}^{2}
$$


or

$$
r_{v i r}=\frac{V_{v i r}}{\sqrt{\frac{1}{2}\left(\Delta_{c}-\Omega_{\Lambda}\right)} H(z)}
$$

Similarly, we can now rewrite the halo mass as

$$
M=\frac{V_{v i r}^{2} r_{v i r}}{G}=\frac{V_{v i r}^{3}}{G H(z) \sqrt{\frac{1}{2}\left(\Delta_{c}-\Omega_{\Lambda}\right)}}
$$

Assuming the matter which settles in the disk to be some fraction $m_{d}$ of the halo mass and the angular momentum of this settling matter to be some fraction $j_{d}$ of the halo's angular momentum, a straightforward derivation (e.g., Mo et al. 1998a) allows one to obtain

$$
M_{d}=\frac{m_{d} V_{v i r}^{3}}{G H\left(z_{f}\right) \sqrt{\frac{1}{2}\left(\Delta_{c}-\Omega_{\Lambda}\right)}}
$$

for the mass of the disk and

$$
R_{d}=\frac{1}{\sqrt{2}}\left(\frac{j_{d}}{m_{d}}\right) \lambda r_{v i r}
$$

for the radius of the disk. The dimensionless angular momentum parameter $\lambda$ is defined as

$$
\lambda=J|E|^{1 / 2} G^{-1} M^{-5 / 2}
$$

where $J$ is angular momentum, $M$ is the mass, and $E$ is the total energy of the bound system.

There are three relatively simple reasons to go beyond this simple approach. First, the adiabatic contraction of the halo due to dissipation of baryons towards the halo center will modify the halo profile. Second, numerical simulations show that model halos actually have a Navarro, Frenk, \& White (1997) profile for the the dark halo rather than the isothermal profile used above. Finally, in order to make comparisons back to the observations, it is important to consider the observationally-measured rotational velocities of the disk rather than the rotational velocities of the halo proper. Mo et al. (1998a) have found approximate fitting formulas for the consequent corrections made to the disk radius $R_{d}$ and the circular velocity at $3 R_{d}$ :

$$
\begin{aligned}
& R_{d}= \frac{1}{\sqrt{2}}\left(\frac{j_{d}}{m_{d}}\right) \lambda r_{v i r} f_{c}^{-1 / 2} f_{R} \\
& V_{c}\left(3 R_{d}\right)=V_{v i r} f_{V}
\end{aligned}
$$

where approximate fitting functions for $f_{c}, f_{R}$, and $f_{V}$ are given by

$$
f_{c} \approx \frac{2}{3}+\left(\frac{c}{21.5}\right)^{0.7}
$$




$$
\begin{aligned}
f_{R} & \approx\left(\frac{\lambda}{0.1}\right)^{-0.06+2.71 m_{d}+0.0047 / \lambda}\left(1-3 m_{d}+5.2 m_{d}^{2}\right)\left(1-0.019 c+0.00025 c^{2}+0.52 / c\right) \\
f_{V} & \approx\left(\frac{\lambda}{0.1}\right)^{-2.67 m_{d}-0.0038 / \lambda+0.2 \lambda}\left(1+4.35 m_{d}-3.76 m_{d}^{2}\right) \frac{1+0.057 c-0.00034 c^{2}-1.54 / c}{[-c /(1+c)+\ln (1+c)]^{1 / 2}}
\end{aligned}
$$

where $c$ is the standard halo concentration parameter for the Navarro et al. (1997) profile. For simplicity, we use $m_{d}=0.05$ and $c=10$ to convert $V_{v i r}$ to $V_{c}$ in order to compare with the observations. We do not use Eqs. (15)-(16) for these comparisons. Note that larger values of $m_{d}$ render disks unstable at relatively faint surface brightnesses and thus have difficulty accounting for Freeman Law-type surface brightnesses (Freeman 1970).

On the basis of these simple halo scaling relations, the size and mass of disks at any redshift simply scales as $1 / H\left(z_{f}\right) \sqrt{\frac{1}{2} \Delta_{c}\left(z_{f}\right)-\Lambda\left(z_{f}\right)}, z_{f}$ being the redshift at which these high-redshift disks formed. Consequently, the size and luminosity scale as

$$
\begin{gathered}
r(z)=\frac{V_{v i r}}{\sqrt{\frac{1}{2}\left(\Delta_{c}\left(z_{f}(z)\right)-\Omega_{\Lambda}\left(z_{f}(z)\right)\right)} H\left(z_{f}(z)\right)} \\
L(z)=\frac{L}{M}(z) M(z)=\frac{L}{M}(z) \frac{V_{v i r}^{3}}{G H\left(z_{f}(z)\right) \sqrt{\frac{1}{2}\left(\Delta_{c}\left(z_{f}(z)\right)-\Omega_{\Lambda}\left(z_{f}(z)\right)\right.}} f_{V}
\end{gathered}
$$

Using Eq. (3) and (4), we now have our functions $E_{R}(z)$ and $E_{L}(z)$ :

$$
\begin{gathered}
E_{R}(z)=\frac{H\left(z_{f}(0)\right) \sqrt{\frac{1}{2}\left(\Delta_{c}\left(z_{f}(0)\right)-\Omega_{\Lambda}\left(z_{f}(0)\right)\right)}}{H\left(z_{f}(z)\right) \sqrt{\frac{1}{2}\left(\Delta_{c}\left(z_{f}(z)\right)-\Omega_{\Lambda}\left(z_{f}(z)\right)\right)}} \\
E_{L}(z)=\frac{\gamma(0) H\left(z_{f}(0)\right) \sqrt{\frac{1}{2}\left(\Delta_{c}\left(z_{f}(0)\right)-\Omega_{\Lambda}\left(z_{f}(0)\right)\right)}}{\gamma(z) H\left(z_{f}(z)\right) \sqrt{\frac{1}{2}\left(\Delta_{c}\left(z_{f}(z)\right)-\Omega_{\Lambda}\left(z_{f}(z)\right)\right)}}
\end{gathered}
$$

where $\gamma(z)$ is the mass-to-light ratio at redshift $z$. Note that this is quite different from scaling these disks simply in terms of the redshift at which these disks were observed, particularly in the case of low $\Omega$ where little evolution in the size or baryonic mass of the disk population is expected.

We assume that the $z=0$ luminosity function scales in number as a function of $z$ in an analogous way to how the $10^{12} M_{\odot}$ halos scale in number. Using the Press-Schechter (Press \& Schechter 1974) mass function

$$
N(M, z) d M=-\sqrt{\frac{2}{\pi}} \frac{\rho_{0}}{M} \frac{\delta_{c}}{\sigma(M) D(z)} \exp \left(-\frac{\delta_{c}^{2}}{2 \sigma^{2}(M) D(z)^{2}}\right) \frac{d \sigma(M)}{d M} d M
$$


we see that the halo number density scales as

$$
n \propto[D(z)]^{-1}
$$

since the exponential factor remains approximately unity for the $10^{12} M_{\odot}$ mass scale. In terms of the formalism of Eqs. (3-5),

$$
E_{N}(z)=\frac{D(0)}{D(z)}
$$

Here, $D(z)$, the growth factor, was computed using the formula tabulated in Carroll, Press, \& Turner (1992).

We now provide a theoretical and observational justification for our size-luminosity distribution. Theoretically, in the Fall \& Estathiou (1980) picture, the spread in surface brightnesses derives from the spread in dimensionless angular momenta for halos. An approximate parameterization of the dimensionless angular momentum distribution is

$$
p(\lambda)=\frac{1}{\sqrt{2 \pi \sigma_{\lambda}}} \exp \left[-\frac{\ln (\lambda / \bar{\lambda})^{2}}{2 \sigma_{\lambda}^{2}}\right] \frac{d \lambda}{\lambda}
$$

For $\bar{\lambda}=0.05$ and $\sigma_{\lambda}=0.5$, the above expression closely approximates the distribution obtained from N-body simulations (Warren et al. 1992; Cole \& Lacey 1996; Catelan \& Theuns 1996) and analytical treatments (Steinmetz \& Bartelmann 1995). The above spread in dimensionless angular momentum directly translates into the following distribution of sizes:

$$
\phi(r) d r=\frac{1}{\sqrt{2 \pi \sigma_{\lambda}}} \exp \left[-\frac{\ln \left(r / \bar{r}_{e}\right)^{2}}{2 \sigma_{\lambda}^{2}}\right] d \log r
$$

For disks with a constant mass-to-light ratio, it follows from Eqs. (13-16) that $r_{d} \propto r_{v i r} \propto V_{v i r} H\left(z_{f}\right)^{-1} \propto M_{d}^{1 / 3} H\left(z_{f}\right)^{-2 / 3} \propto L_{d}^{1 / 3} H\left(z_{f}\right)^{-2 / 3}$. Ignoring the dependence of $H\left(z_{f}\right)^{-2 / 3}$ on the luminosity, it follows that the surface brightness $\left(L_{d} / r_{d}^{2}\right)$ scales as $L_{d}^{1 / 3}$.

In fact, de Jong \& Lacey (1998) found that the Mathewson, Ford, \& Buchhorn (1992) data set gave a good fit to the following bivariate size-luminosity distribution with similar properties to those predicted above:

$$
\begin{gathered}
\Phi\left(r_{e}, M\right) d \log r_{e} d M=\frac{\Phi_{0}}{\sigma_{\lambda} \sqrt{2 \pi}} \exp \left(-\frac{1}{2}\left[\frac{\log r_{e} / r_{e}^{*}-0.4\left(M-M_{*}\right)(2 / \beta-1)}{\sigma_{\lambda} / \ln (10)}\right]^{2}\right) \\
10^{-0.4 *\left(M-M_{*}\right)(\alpha+1)} \exp \left(-10^{-0.4 *\left(M-M_{*}\right)}\right) d \log r_{e} d M
\end{gathered}
$$

where $\Phi_{0}=0.0033 \mathrm{Mpc}^{-3}, \alpha=-1.04, \beta=3, M_{*}=-22.8, r_{e}^{*}=7.9 \mathrm{kpc}$, and $\sigma_{\lambda}=0.37$ (converting their sizes and luminosities from $h_{0}=0.65$ to the $h_{0}=0.50$ used here). Implicit 
in the above bivariate distribution is a distribution in sizes analogous to Eq. (31), a Schechter distribution in luminosity, and a $S B \propto L^{1 / 3}$ correlation between luminosity and surface brightness. Similar scalings are apparent in the McGaugh \& de Blok (1997) sample.

Now let us compare a typical $L_{*}$ galaxy in this model with the observations. Using our stated assumption, a $L_{*}$ galaxy has a mass of $1.1 \cdot 10^{12} M_{\odot}$. A typical formation time occurs at $z=0.3$. Using Eqs. (13-14), the circular velocity and size of the halo is $132 \mathrm{~km} / \mathrm{s}$ and $272 \mathrm{kpc}$ (compared to the $140 \mathrm{~km} / \mathrm{s}$ and $241 \mathrm{kpc}$ predicted assuming a constant $200 \rho_{c}$ for the collapse density as in Mo et al. 1998). Then, using Eq. (18), the size of the disk is $\sim 6.0 \mathrm{kpc}$.

This is smaller than the empirical findings of de Jong \& Lacey (1998) (7.9 kpc), our

own comparisons to local observations (\$4.4) (6.9 kpc), and Freeman's Law, which gives 6.9 kpc. Supposing this to be due to a slight cut-off at low values of the dimensionless angular momentum due to disk instabilities (Efstathiou, Lake \& Negroponte 1982; Dalcanton et al. 1997; Mo et al. 1998a; van den Bosch 1998), we scale up the size of a typical $L_{*}$ disk galaxy to $6.9 \mathrm{kpc}$ and reduce the spread in dimensionless angular momenta to $\sigma_{\lambda}=0.37$, as found by de Jong \& Lacey (1999) in the analysis of the Mathewson et al. (1992) sample.

Throughout our analysis, we shall take the $\Omega=0.3, \Omega_{\Lambda}=0.7$ model as our preferred fiducial hierarchical model because of its better correspondence with the evolution in the number of small disks observed up to $z \sim 1$ (Mao et al. 1998). We evolve the mass-to-light ratio $\gamma(z)$ as $(1+z)^{-0.5}$ to reproduce the observed evolution in the Tully-Fisher relationship (see $\S 4.2)$.

\subsection{Infall Model (Backwards Approach)}

Instead of trying to determine how the global structural properties of disks evolve based on the corresponding properties of their ambient halos, it is also possible to examine a number of local disk galaxies in great detail and to use detailed models of their observed properties (gas profiles, stellar profiles, metallicity profiles, current SFR profiles, age-metallicity relationships) to determine how galaxies might have evolved to high redshift.

There is no consideration of how individual halos might evolve backwards in time in these models, both for simplicity and because of large uncertainties in the local distribution of dark matter. Consequently, while for the forwards approach, the entire evolution of disk properties derives from an evolution of the halo properties, the infall models considered here completely ignore these effects. Conversely, while the forwards approach presented here ignores issues related to the manner in which halo gas is converted into stars, for the 
infall model, such issues are important.

Naturally, given that one always adopts the observed local universe in this approach as known, this approach does not explain in and of itself why the local disk population is as it is. Indeed, it cannot since there is no link to the initial conditions. In this view, for the hierarchical approach, we adopted the local universe we did because it was a natural prediction of the model, and for the infall approach, we adopted it because it agrees with the observations.

We examine such a model for the evolution of local galaxies based upon the Prantzos \& Aubert (1995) model for the star formation rates, metallicites, stars, and gas content for the Milky Way disk. We previously presented this infall model elsewhere (Bouwens et al. 1997; Cayón et al. 1996), and we shall revisit it here. This model ignores radial inflows for simplicity and takes the star formation rate to be proportional to both the gas surface density $\left(\Sigma_{g}\right)$ and the reciprocal of the radius $r$, which for a flat rotation curve is proportional to the epicyclic frequency:

$$
\frac{d \Sigma_{*}(r, t)}{d t}=\frac{\Sigma_{g}(r, t)}{\tau_{g}(r)}
$$

where $\tau_{g}(r)=\left[0.3\left(r / r_{\odot}\right)^{-1} \mathrm{Gyr}\right]^{-1}$. Physically, such a star formation rate results if the star formation rate is proportional to the rate at which molecular clouds collide (Wang \& Silk 1994) or the periodic compression rate (Wyse \& Silk 1989).

For simplicity, the accretion time scale $\tau_{f f}$ was taken to be independent of radius since a variation in this time scale is not strongly constrained by the observations (Prantzos \& Aubert 1995). The spread in Hubble types was then naturally taken to arise from a spread in this time scale (Cayón et al. 1996). The equation for the evolution of the gas density is then

$$
\frac{d \Sigma_{g}(r)}{d t}=\frac{\Sigma_{g}(r, T)+\Sigma_{*}(r, T)}{1-e^{-T / \tau_{f f}}} \frac{e^{-t / \tau_{f f}}}{\tau_{f f}}-\frac{\Sigma_{g}(r)}{\tau_{g}}
$$

where $T$ is the time from the formation of the disk to the present. Integrating these equations yields the result

$$
\Sigma_{g}(r, t)=\frac{\Sigma_{g}(r, T)+\Sigma_{*}(r, T)}{1-e^{-T / \tau_{f f}}} \frac{e^{-t / \tau_{f f}}-e^{-t / \tau_{g}}}{\tau_{f f}-\tau_{g}} \tau_{g}
$$

Given the fact that this model derived from only one galaxy, it is difficult to know how to extend its evolutionary predictions to galaxies with different luminosities and surface brightnesses. One possible means of extending this model to galaxies beyond the Milky Way involves simply scaling the star formation rates by the differential rotation rate. The change 
in the star formation rate would then be proportional to $V_{c} / R$ or the typical dynamical time for the disk. Since $R$ is roughly proportional to $V_{c}$, there would be no large change in the time scales as a function of disk mass or rotational velocity. Accordingly, Bouwens et al. (1997) simply elected to scale everything in size to reproduce all luminosities while conserving surface brightness, which is precisely what we have done here.

To determine the scaling relations for galaxies using the infall approach, we performed the calculation for each galaxy on a series of 30 different rings varying logarithmically in size, where the smallest is a circle with radius $0.2 \mathrm{kpc}$ and the largest is a ring of radius $60 \mathrm{kpc}$ with width $12 \mathrm{kpc}$ as done in Bouwens et al. (1997) and Roche et al. (1998). We calculate the evolution in rest-frame $B$ band magnitudes by evolving each ring separately to keep track of its gas mass, stellar composition, and metallicity, and we output its colors using the Bruzual \& Charlot instantaneous-burst metallicity-dependent spectral synthesis tables compiled in Leitherer et al. (1996).

In the rest-frame $B$ band, we found the following scaling relationships:

$$
\begin{gathered}
E_{L}(z)=10^{-0.4(-0.6 z)} \\
E_{R}(z)=1-0.27 z
\end{gathered}
$$

Clearly, without number evolution, we take $E_{N}(z)=1$.

For the sake of clarity, we note that the present model differs from the one presented in Bouwens et al. (1997) in terms of both the luminosity functions used and the bulge-to-total distribution assumed. Furthermore, in the Bouwens et al. (1997) study, the preferred values of the age $T$ and gas-infall time scale $\tau_{f f}$ used in the Prantzos \& Aubert (1995) study were scaled to reproduce the number counts. No such scaling was attempted in the present model and we simply use the same $\tau_{f f}$ for all disk types.

This model is similar in spirit to the size-luminosity evolution model presented by Roche et al. (1998) based on the infall models of Chiappini et al. (1997), which models the infall, star formation, and chemical evolution of both the thin and thick disk components. For the purposes of illustration, we shall compare the Chiappini et al. (1997) infall model to the one just described in $\S 4.1$, after which we will restrict our consideration to the redshift scalings given by the Prantzos \& Aubert prescription. In this model, the star formation time scale is equal to

$$
\tau= \begin{cases}1 \mathrm{Gyr}, & r<2 \mathrm{kpc} \\ (0.875 r-0.75) \mathrm{Gyr}, & r \geq 2 \mathrm{kpc}\end{cases}
$$

The star formation commences at $t_{\text {form }}=(16 \mathrm{Gyr}-0.35 \tau)$. Note that the time scale for star formation here depends on the radius to the first power as in our model, the preferred Prantzos \& Aubert (1995) model, and the recent work by Boissier \& Prantzos (1999). 


\section{Observations}

\subsection{Low-Redshift Samples}

We make comparisons against local samples with information on the size, luminosity, and circular velocity of local galaxies. Though in principle we could have just used the Courteau (1997) sample, we follow Mao et al. (1998) in using a compilation of three different samples for the comparisons which follow to examine the three two-dimensional relationships.

\section{de Jong $\mathscr{G}$ van der Kruit Sample:}

The de Jong \& van der Kruit (1994) sample provides a nice sample for examining the local size/magnitude relationship. It is selected from the Uppsala Catalogue of Galaxies (Nilson 1973, hereinafter UGC), over only $\sim 12.5 \%$ of the sky and uses only relatively face-on $(b>0.625)$ galaxies (37.5\% of all orientations). Following de Jong (1996), we also take it to be diameter-limited in $R$ to galaxies larger than $2^{\prime}$ at $24.7 R$-band mag $/ \mathrm{arcsec}^{2}$. Whereas de Jong \& van der Kruit (1994) in their treatment of their sample assume transparent disks, we correct observed magnitudes to an inclination of $70 \mathrm{deg}$ using the Tully \& Fouqué (1985) inclination corrections.

\section{Courteau (1997) Sample:}

We use the Courteau (1997) sample to calibrate the local $z=0 V_{c}$ - size relationship. The Courteau (1997) sample contains 304 Sb-Sc galaxies from the UGC with Zwicky magnitudes $m_{B}<14.5, R$-band angular diameters larger than $1^{\prime}$, and $B$-band major axis $<4^{\prime}$. We take their $v_{\text {opt }}=V_{c}\left(3.2 R_{d}\right)$ as the circular velocity and the $25 \mathrm{rmag} / \mathrm{arcsec}^{2}$ isophote as the radius.

Pierce \& Tully (1988) Sample:

We use the Pierce \& Tully (1988) sample to calibrate the local $z=0 V_{c}$ - luminosity relationship. The Pierce \& Tully sample was taken from galaxies in the area of the Ursa Major cluster and is complete up to $B_{T}<13.3$. It includes all galaxies which are not elliptical or S0, not more face on than $30 \mathrm{deg}$, and not possessing confused H I profiles. Note that in this study and in the Vogt et al. $(1996,1997)$ studies to be discussed, the observed absolute $B$-band magnitudes were corrected to intrinsic (unextincted) values using the Tully \& Fouqué (1985) inclination corrections. 


\subsection{High-Redshift Samples}

Simard et al. (1999) Sample:

For the magnitude-radius relationship, we use the data presented by Simard et al. (1999). This data set contains structural information for $\sim 200$ galaxies to $I<23.5$ from 6 different WFPC2 pointings in the Groth strip $\left(\sim 30 \operatorname{arcmin}^{2}\right)$. Spectra were obtained for only a fraction of the faint galaxies, but for galaxies with spectra, there was nearly $100 \%$ redshift identification. Following Simard et al. (1999), we can quantify the selection effects of this sample. The probability that a galaxy with apparent magnitude $I_{814}$ and radius $r_{d}$ would fall in the photometric sample is $S_{U P}\left(I_{814}, r_{d}\right)$. From Figure 4 of Simard et al. (1999), we have approximated this as

$$
\begin{cases}1, & I_{814}+5 \log r_{d}(\prime \prime)<21 \\ 1-\frac{2}{3}\left(I_{814}+5 \log r_{d}(\prime \prime)\right), & 21<I_{814}+5 \log r_{d}(\prime \prime)<22.5 \\ 0, & 22.5<I_{814}+5 \log r_{d}(\prime \prime)\end{cases}
$$

(Actually, this provides a steeper surface brightness cut-off than the selection function given in Simard et al. 1999.) The probability that a galaxy with apparent magnitude $I_{814}$ and radius $r_{d}$ would be selected from the photometric sample for spectroscopic follow-up is given by $S_{P S}\left(I_{814}, r_{d}\right)$, which we have approximated as

$$
\begin{cases}1, & I_{814}<19.3 \\ 1-\frac{0.8}{4}\left(I_{814}-19.3\right), & 19.3<I_{814}<23.5 \\ 0, & 23.5<I_{814}\end{cases}
$$

(again by eyeballing Figure 4 of Simard et al. 1999). Putting these two selection effects together, the probability of selecting a galaxy with apparent magnitude $I_{814}$ and radius $r_{d}$ is simply the product of these quantities, namely, $S_{U P}\left(I_{814}, r_{d}\right) S_{P S}\left(I_{814}, r_{d}\right)$. Given our ignorance about the inclinations used in the Simard et al. (1999) study, we assume an average inclination of $60 \mathrm{deg}$ in transforming the absolute magnitudes to an inclination of 70 deg using the Tully \& Fouqué (1985) law, which yields a correction of $0.27^{m}$ here.

\section{Lilly et al. (1998) Sample:}

We also use the data from the LDSS2-CFRS sample with HST WFPC2 follow-up to look at the magnitude-radius relationship. From Table 3 of Brinchmann et al. (1998), the effective area of the CFRS portion of this sample is $0.01377 \mathrm{deg}^{2}\left(49 \operatorname{arcmin}^{2}\right)$. The survey is magnitude limited to $17.5<I<22.5$, where the magnitudes are isophotal to $28.0 I_{A B} \mathrm{mag} / \operatorname{arcsec}^{2}$. Though the surface brightness limit is quoted as $24.5 I_{A B} \mathrm{mag} / \operatorname{arcsec}^{2}$, we have used the more conservative surface brightness limit $23.5 I_{A B} \mathrm{mag} / \operatorname{arcsec}^{2}$. In 
principle, then, for any galaxy detected the isophotal magnitude should be approximately equal to the total magnitude. Lilly et al. (1998) chose to examine that subset of galaxies from this data-set which were disk-dominated and had disk scale lengths $>4 \mathrm{kpc}$, a sample we shall henceforth refer to as the Lilly et al. (1998) large disk sample. Given that the central surface brightness is not strongly correlated with inclination angle, Lilly et al. (1998) concludes that disks are consistent with being opaque. We use the Tully \& Fouqué (1985) to transform the listed absolute magnitudes to an inclination of $70 \mathrm{deg}$.

Vogt et al. (1997) Sample:

In contrast to high-redshift samples with both magnitude and radial information, high-redshift samples with circular velocity measurements are considerably smaller and possess less well-defined selection criteria. In fact, the Vogt et al. (1996,1997) sample with 16 galaxies is the largest such published sample. The selection criteria for this sample is still somewhat qualitative and patchy in nature. It considers galaxies with an inclination greater than $30 \mathrm{deg}$, detectable line emission, undistorted disk morphology, and an extended profile. We assume an $I_{814}<22.5$ magnitude limit as used in the Vogt et al. (1997) sample.

\section{Results}

\subsection{Basic Scalings}

Before getting into a detailed comparison of the models with the observations, we begin by illustrating the manner in which the sizes of $L_{*}\left(M \sim 1.2 \cdot 10^{12} M_{\odot}\right)$ galaxies typically evolve as a function of redshift for the different models in Figure 1 . We present this evolution in terms of the rest-frame $B$ half-light radius, the specification of a band and a measure being necessary to the intrinsic band and profile dependence of evolution in the infall model.

The hierarchical models predict more size evolution than expected from both infall models considered here. Of course, the $\Omega=1$ model possesses more size evolution than the $\Omega=0.3 ; \Omega_{\Lambda}=0.7$ model, and the $\Omega=0.3 ; \Omega_{\Lambda}=0.7$ model more size evolution than the $\Omega=0.1$ model because of the steeper dependence of $1 / H(z)$ at the typical formation

redshifts of disks to $z \sim 1$. The predictions of both the Prantzos \& Aubert (1995) and Chiappini et al. (1997) models are quite similar, encouragingly enough given that Prantzos \& Aubert (1995) and Chiappini et al. (1997) models represent completely independent efforts to model the evolution of the Milky Way galaxy.

Taking $R / V_{c}$ as the measure of size for a given mass halo, we plot both the Courteau 
sample at $z=0$ and the higher-redshift data of Vogt et al. (1996,1997) scaled appropriately so that the Courteau data is centered on unevolved scale size of a galaxy at $z=0$. At face value, a comparison of the Vogt data with the Courteau data indicates that there has been size evolution from $z=0$ to $z=1$ as any of the models here would predict (Mao et al. 1998). Nevertheless, the lack of a strong trend in redshift across the Vogt data-set makes one suspicious that there may be selection effects at work or even systematic errors in the measurements of parameters which may produce the observed differences. In any case, strong conclusions must await the compilation of a larger high-redshift dataset, where the selection effects have been more carefully quantified.

We also present model scalings of the number, luminosity, and surface brightness

expected for $L_{*}$ galaxies in Figure 1. By construction, the hierarchical models produce more number evolution than the infall models, which involve no evolution in number. Except for the infall model based upon the Chiappini et al. (1997) prescription, our "Infall" model produces similar evolution in luminosity as the hierarchical models to $z \sim 1$. The infall models also produce less surface brightness evolution than the hierarchical models.

\subsection{Tully-Fisher Relationship}

To assess hierarchical and infall models, we compare their predicted Tully-Fisher relationships with both low and high-redshift observations in the left panels of Figures 2-3. For the Monte-Carlo simulations, we use the same selection effects as already specified for the low (Pierce \& Tully 1988) and high-redshift (Vogt et al. 1996, 1997) samples. We have added the Pierce \& Tully (1992) fit to these plots for comparison. Naturally, for both our hierarchical and infall models, we obtained good agreement with the Pierce \& Tully (1988) sample since we used that sample to adjust the mass-to-light ratio (we assumed that a $M_{b_{J}}=-21$ galaxy had a mass $1.2 \cdot 10^{12} M_{\odot}$ ) and its assumed log-normal scatter (one sigma scatter of 0.3 dex). At higher redshift, we again obtain basic agreement with the Vogt et al. $(1996,1997)$ sample for both our infall and hierarchical models.

\subsection{Size- $V_{c}$ Relationship}

We also compare our model predictions with the low and high-redshift observations for the size- $V_{c}$ relationship in the right panels of Figures 2-3. Again, we apply the selection criteria given in $\S 3$ to the low (Courteau 1997) and high-redshift (Vogt et al. 1996,1997) model results. At low redshift, we obtain basic agreement with the observations of Courteau 
(1997) though there seems to be a slight shift in our models toward larger sizes. No adjustment of our models was made to obtain agreement with the Courteau (1997) sample, and so this comparison can be considered a self-consistency check on our $z=0$ models.

At high redshift, sizes for both models are consistent, if not a little larger than the sizes in the Vogt et al. $(1996,1997)$ sample. One of the most surprising thing about a comparison of the models with the observations is the significant size evolution observed in the lowest redshift bin $(z<0.6)$ relative to the models. In fact, as discussed in relation to Figure 1, the low redshift $(z<0.6)$ points seem to have undergone more size evolution than the high redshift $(z>0.6)$ points. As the low redshift points are primarily low luminosity galaxies and the high redshift high luminosity galaxies, this could point to some luminosity-dependent evolutionary trend though the numbers are still too small to make any claims toward this end.

\subsection{Size-Magnitude Relationship}

As so often in making low-to-high redshift comparisons, freedom in the choice of the $z=0$ no-evolution model can be very important in interpreting the high-redshift results. In particular, due to the fact that each redshift bin in the Simard et al. (1999) sample contains galaxies of a particular luminosity, significant evolution in disk surface brightness would appear to be present, simply as a result of correlations between luminosity and surface brightness at $z=0$. There are also important surface brightness selection effects in constructing the Simard et al. (1999) sample. We illustrate the importance of these considerations in Figure 4 by presenting a no-evolution model, hereafter referred to as our fiducial no-evolution model, identical to our $z=0$ hierarchical and infall models except there is no-evolution in the disk size, number, surface brightness, or luminosity, i.e., $E_{L}(z)=E_{N}(z)=E_{R}(z)=1$. We also present the surface brightness distributions recovered from a similar no-evolution model differing only in its use of the $M_{b_{J}}=-21$ surface brightness distribution for all luminosities. We also present the surface brightness distributions recovered both by including a less conservative surface brightness selection

$$
S_{U P}\left(I_{814}, r_{d}(\prime \prime)\right)= \begin{cases}1, & I_{814}+5 \log r_{d}(\prime \prime)<21 \\ 1-\frac{1}{3}\left(I_{814}+5 \log r_{d}(\prime \prime)\right), & 21<I_{814}+5 \log r_{d}(\prime \prime)<24 \\ 0, & 24<I_{814}+5 \log r_{d}(\prime \prime)\end{cases}
$$

(more resembling the one used by Simard et al. (1999)) and without including surface brightness $\left(S_{U P}\right)$ selection at all. Notice the apparent increase in surface brightness for our fiducial model, where $S B \propto L^{1 / 3}$, as a function of z relative to our constant surface 
brightness model. It is interesting to note that there is an absence of galaxies at surface brightnesses close to the threshold for detection in the Simard et al. (1999) sample.

With these caveats in mind, we compare our model $B$-band surface brightness distributions with both the low and high redshift observations in Figure 5. The models seem to be in rough agreement with the surface brightness distribution of the observations. Given that model populations increase in $B$-band surface brightness by $\sim 1.5^{m}$ to $z \sim 1$ (see Figure 1), this suggests a similar increase in the surface brightness of disks to $z \sim 1$. The models themselves show no large differences. As so often, the uncertainties in the $z=0$ modeling are large enough to preclude detailed discrimination among models, especially given the limited high redshift data sets.

In Figures 6-7, we plot the observed luminosity-size distributions at low (de Jong \& van der Kruit 1994) and high (Simard et al. 1999) redshift and compare them with those obtained for the $\Omega=0.3 / \Omega_{\Lambda}=0.7$ hierarchical and infall models. We show a similar comparison of the hierarchical model with the high redshift Lilly et al. (1998) large disk data-set in Figure 8. We also plot the cumulative size and luminosity distributions along the vertical and horizontal axes, respectively, for both the observations (histogram) and the models (lines).

As in Figures 4-5, the models predict too many low surface brightness galaxies relative to the observations. This also results in too many large model galaxies and too many low luminosity model galaxies relative to the observed size and luminosity distributions at intermediate to low luminosities, especially for the Lilly et al. (1998) large disk sample even with our more conservative surface brightness limit. For large, luminous galaxies, there is no obvious change in numbers to high redshift.

In Figures 6-7, there are also relatively large differences in normalization between the observations and models. This is apparently the result of large-scale structure (there are small groups/clusters at $z \sim 0.8$ and $z \sim 1.0$ in the Groth Strip). It is therefore difficult to make extremely quantitative statements about the evolution in the number density of galaxies with specific surface brightnesses, sizes, and luminosities across the redshift intervals surveyed.

Nevertheless, the abundance of high surface brightness disk galaxies at high redshifts relative to the model predictions is surely conspicuous and suggests that there has been a significant increase in the number of high surface brightness disk galaxies to $z \sim 1$ as we have already argued in comparing the model and observed surface brightness distributions. Again, shifting the surface brightness distribution toward these high surface brightnesses (here by the $\sim 1.5^{\mathrm{m}}$ predicted by the models) is an obvious way of accommodating this 
increase. Of course, any argument based on the normalization of specific galaxy populations is subject to considerable uncertainties important when such small contiguous areas are being probed.

To provide a visual comparison between our no-evolution and evolutionary models, we include a simulation of a patch of the $\operatorname{HDF}\left(I_{F 814 W}, B_{F 450 W}\right.$, and $\left.V_{F 606 W}\right)$ in Figure 9 using our fiducial no-evolution model (panel a), the $\Omega=1$ hierarchical model (panel b), and the $\Omega=0.3, \Omega_{\Lambda}=0.7$ infall model (panel c) for comparison with the actual HDF North and South (panel d). Clearly, the lack of high surface brightness galaxies is apparent in the no-evolution model relative to the HDF and even somewhat in the apparent in the $\Omega=1$ hierarchical model relative to the HDF. Of course, our simulations do not include ellipticals or peculiars, so the actual HDF will include more bright objects than the simulations.

\section{Discussion}

There is a real question about a lack of low surface brightness galaxies relative to our predictions, especially as compared to the no-evolution model predictions. This conclusion is somewhat dependent on the assumed correlation between surface brightness and luminosity as is evident in Figure 4. This conclusion is also dependent on the selection biases against low surface brightness galaxies not being stronger than those considered here.

There is an extensive literature discussing surface brightness selection biases (Disney 1976; Allen \& Shu 1979) and various attempts to derive the bivariate luminosity-surface brightness distribution of galaxies (McGaugh 1996; Dalcanton et al. 1997b; Sprayberry et al. 1997). Surface brightness has a particularly strong effect on isophotal magnitude determinations, especially for low surface brightness galaxies; and this can introduce significant errors in the magnitude determinations, so the effective volume probed for these galaxies is significantly smaller than it is for equivalent luminosity high surface brightness galaxies (McGaugh 1996).

Simard et al. (1999) in a detailed quantification of the selection effects of the DEEP sample do not consider the effect of surface brightness on the magnitudes and sizes recovered since typical errors were found to be $0.2^{m}$ (Simard 1999, private communication). Despite the relatively small size of this error, it is not entirely clear to the present authors that the errors would not become quite significant for the lowest surface brightness galaxies in the sample, particularly those just marginally detectable given the chosen object identification and photometric parameters. Secondly, Simard et al. (1999) considers disk galaxies to be

optically thin whereas the observations of Lilly et al. (1998) are more consistent with disks 
being optically thick. Highly-inclined optically thin disks would be much more detectable than face-on or optically-thick disks. The upshot is that at many apparent magnitudes and radii, Simard et al. (1998) would suppose that at least some highly inclined galaxies would be detectable and therefore the selection function $S_{U P}$ there would be non-zero when in reality if disks were optically thick it would be zero. For these reasons, we used a slightly more conservative selection function in surface brightness than that given in Figure 4 of Simard et al. (1999) (see §3.2).

Another possibility, not considered here, is that low surface brightness galaxies might form relatively late, meaning that their mass-to-light ratios remain relatively large until relatively recent epochs. Of course, prima facie, this would seem unlikely given the apparently constant slope in the Tully-Fisher relationship to faint magnitudes.

In their own analysis of their sample of $\sim 200$ galaxies, Simard et al. (1999) concluded that there had been little evolution in the surface brightness distribution of disk galaxies when all selection effects had been carefully considered, quite in contrast to our estimated $\sim 1.5^{m}$ of $B$-band surface brightness evolution. Little consideration, however, was paid to the evolution in the total numbers of high surface brightness galaxies. Here, we find that the number of high surface brightness galaxies dramatically exceeds that predicted by the evolutionary models considered here, and we have argued that this provides evidence for an evolution in the surface brightness distribution of disk galaxies.

Our interpretation seems to be furthermore supported by the lack of low surface brightness galaxies relative to our models. For no-evolution in the disk surface brightness distribution really to be present as Simard et al. (1999) claims, high-redshift intervals should have similar numbers of low surface brightness galaxies to those found in local samples, and these galaxies seem to be deficient, even with respect to our models which show significant evolution in surface brightness.

While the conclusions of Simard et al. (1999) appear to have been carefully drawn, we would like to suggest that there are significant uncertainties in their determination of the mean surface brightnesses in the lowest redshift intervals and therefore the inferred evolution in surface brightness due to the small size of the low redshift samples considered. By applying the selection effects from the high-redshift bin identically to all redshift intervals, Simard et al. (1999) restricted their analysis to that fraction of disk galaxies exceeding the high-redshift surface brightness detection limit. Applying these selection criteria uniformly to all low redshift intervals severely pares down the low-redshift samples and significantly increases the uncertainty of their average surface brightness measure. Given the observed range in observed surface brightness $\left(\sim 2 \mathrm{mag} / \mathrm{arcsec}^{2}\right)$ and typical numbers $(\sim 5-6)$ for the lowest redshift bins, there is a non-negligible uncertainty in the 
average surface brightness at low redshift, $\sim 0.6^{m}$.

Our estimates of $\sim 1.5^{\mathrm{m}}$ of $B$-band surface brightness evolution are somewhat larger than that inferred by most authors. Roche et al. (1998) found $0.9^{m}$ of surface brightness evolution from $z \sim 0.2$ to $z \sim 0.9$, Lilly et al. (1998) found $0.8^{m}$ of surface brightness evolution in their large disk sample, and Schade et al. $(1995,1996 \mathrm{a})$ inferred $1.2^{m}$ and $1.5^{m}$ respectively to $z \sim 0.8$. Despite different differential measures of surface brightness evolution, most of these samples give similar values for the mean disk surface brightness near $z \sim 1: 20.79 \pm 0.17$ for the Roche et al. (1998) sample $(0.65<z), 19.9 \pm 0.2$ for the Simard et al. (1999) sample $(0.9<z<1.1), 20.7 \pm 0.25$ for the Lilly et al. (1998) large disk sample $(0.5<z<0.75), 20.2 \pm 0.25$ for the Schade et al. (1995) sample $(0.5<z)$, and $19.8 \pm 0.1$ for the Schade et al. (1996) sample $(0.5<z<1.1)$. Consequently, differences in the surface brightness evolution inferred derive from differences in the $z=0$ surface brightness distributions assumed. We assume a distribution consistent with the local data of de Jong \& van der Kruit (1994) and Mathewson et al. (1992) as a baseline for measuring evolution with a surface brightness peaking faintward of Freeman's Law $\left(\sim 21.7 \mathrm{~B}\right.$ mag $/ \mathrm{arcsec}^{2}$; Freeman 1970) while Schade et al. $(1995,1996)$ simply makes reference to Freeman's Law

$\left(\sim 21.65 b_{j} \mathrm{mag} / \operatorname{arcsec}^{2}\right)$. Simard et al. (1999), Lilly et al. (1998), and Roche et al. (1998) measure surface brightness evolution differentially across their samples. Possible problems here are surface brightness selection effects and limited low-redshift samples.

\section{Summary}

In the present paper, we presented models based on two different approaches for predicting the evolution in disk properties: a hierarchical forwards approach, where the evolution in disk properties follows from corresponding changes in halo properties, and a backwards approach, where the evolution in disk properties follows from an infall model providing a close fit to numerous observables for the Milky Way. We normalized the models to the local $z=0$ observations, we made high-redshift predictions for the models, and we compared these predictions with high-redshift observations.

Our findings are as follows:

- The hierarchical and infall models predict relatively similar amounts of evolution in global properties (size, surface brightness, and luminosity) for disk galaxies to $z \sim 1$. Clearly, discriminating between the models will require a careful look at evolution in number (and therefore surveys over a much larger area) and/or measurements of certain internal properties, like color, star formation, or metallicity gradients of high 
redshift disks.

- There is an apparent lack of low surface brightness galaxies in the high-redshift observations of Simard et al. (1999) and Lilly et al. (1998) as compared to model predictions based on local observations (Mathewson et al. 1992; de Jong \& van der Kruit 1994).

- Our model surface brightness distributions produce relatively good agreement with the observations, suggesting that the $B$-band surface brightness has evolved by $\sim 1.5^{\mathrm{m}}$ from $z=0$ to $z \sim 1$ similar to that found in the models. This finding is supported by the fact that there is a significantly larger number of high surface brightness galaxies than in our model predictions, suggesting that there has been a significant evolution in number, most easily accommodated by shifting the mean surface brightness of the disk population to higher surface brightnesses. This is contrary to the conclusion reached by Simard et al. (1999) based on the same data.

Here the hierarchical and infall models were presented as competing models to describe the evolution in the properties of disk galaxies. If the hierarchical structural paradigm is roughly correct as is generally supposed, the infall model simply provides a modification of the basic hierarchical scalings to account for the fact that the gas infall rate or star formation efficiency is not the same at all radii. In this sort of scenario, if there is an appreciable formation of structure at low redshift, a consideration of hierarchical scalings is probably more appropriate and if there is not, a consideration of scalings following from infall models is probably more appropriate. Obviously, at the present time, a complete incorporation of radially dependent gas infall and star formation scenarios into a hierarchical paradigm is not merited given the lack of high-redshift data needed to constrain such hybrid models.

We acknowledge helpful discussions with David Schade and Luc Simard. We are especially grateful to Laura Cayón for helping compile some of the samples used here, for some useful discussions, and for providing a critical read of this document. We thank Stephane Courteau and Nicole Vogt for sending us their data in electronic form. This research has been supported in part by grants from NASA and the NSF. RJB would like to thank the Oxford astrophysics department for its hospitality while this work was being carried out.

\section{REFERENCES}

Allen, R. J. \& Shu, F. H. 1979, ApJ, 227, 67. 
Avila-Reese, V., Firmani, C., \& Hernandez, X. 1998, ApJ, 505, 37.

Bertschinger, E. 1985, ApJS, 58, 39.

Binggeli, B., Sandage, A., \& Tammann, G.A. 1988, ARA\&A, 26, 509.

Boissier, S. \& Prantzos, N. 1999, MNRAS, 307, 857.

Bouwens, R.J., Cayón, L., \& Silk, J. 1997, ApJ, 489, L21.

Bouwens, R.J., Cayón, L., \& Silk, J. 1999, ApJ, in press.

Brinchmann, J., et al. 1998, ApJ, 499, 112.

Bryan, G.L. \& Norman, M.L. 1998, ApJ, 495, 80.

Calzetti, D. 1997, to appear in the Proceedings of the Conference "The Ultraviolet Universe at Low and High Redshift" preprint: astro-ph/9706121.

Carroll, S.M., Press, W.H., \& Turner, E.L. 1992, ARA\&A, 30, 499.

Catelan, P. \& Theuns, T. 1996, MNRAS, 282, 436.

Cayón, L., Silk, J., \& Charlot, S. 1996, ApJ, 467, L53.

Chiappini, C., Matteucci, F., Gratton, R. 1997, ApJ, 477, 765.

Cole, S. \& Lacey, C. 1996, MNRAS, 281, 1176.

Cole, S., Lacey, C.G., Baugh, C.M., Frenk, C.S. 1999 (in preparation).

Contardo, G., Steinmetz, M., \& Fritze-von Alvensleben, U. 1998, ApJ, 507, 497.

Courteau, S. 1997, ApJS, 103, 363.

Dalcanton, J.J., Spergel, D.N., James, J.E., Schmidt, M.,\& Schneider, D.P. 1997, AJ, 114, 635.

Dalcanton, J.J., Spergel, D.N., \& Summers, F.J. 1997, ApJ, 482, 659.

de Jong, R.S. \& van der Kruit, P.C. 1994, A\&AS, 106, 451.

de Jong, R.S. 1996, A\&A, 313, 45.

de Jong, R.S. \& Lacey, C. 1999, preprint.

Disney, M.J. 1976, Nature, 263, 573. 
Efstathiou, G., Lake, G., Negroponte, J. 1982, MNRAS, 199, 1069.

Ferrini, F., Molla, M., Pardi, M.C., \& Diaz, A.I. 1994, ApJ, 427, 745.

Freeman, K. C. 1970, ApJ, 160, 811.

Gunn, J. \& Gott, R. 1972, ApJ, 176, 1.

Lacey, C. \& Cole, S. 1993, MNRAS, 262, 627.

Lacey, C., Cole, S. 1994, MNRAS, 271, 676.

Leitherer et al. 1996, PASP, 108, 996.

Lilly, S., et al. 1998, ApJ, 500, 75.

Matthewson, D.S., Ford, V.L., \& Buchhorn, M. 1992, ApJS, 81, 413.

Mao, S., Mo, H.J., \& White, S.D.M. 1998a, MNRAS, 295, 319.

Mao, S., Mo, H.J., \& White, S.D.M. 1998b, preprint, astro-ph/9807341.

Mao, S., Mo, H.J., \& White, S.D.M. 1998, MNRAS, 297, L71.

McGaugh, S.S. 1996, MNRAS, 280, 337.

McGaugh, S.S., \& de Blok, W.J.G. 1997, ApJ, 481, 689.

Moore, B. et al. 1999, ApJ, 524, 19.

Navarro, J., Frenk, C., \& White, S.D.M. 1997, ApJ, 487, 73.

Navarro, J. and Steinmetz, M. 1999, ApJ, in press.

Nilson, P. 1973, Uppsala General Catalogue of Galaxies (Uppsala: Uppsala Obs. Ann.)

Pozzetti, L., Bruzual, G., \& Zamorani, G. 1996, MNRAS, 274, 832.

Prantzos, N. \& Aubert, O. 1995, A\&A, 302, 69.

Prantzos, N. \& Silk, J. 1998, ApJ, 507, 229.

Press, W. H., \& Schechter, P. L. 1974, ApJ, 187, 425.

Pierce, M.J. \& Tully, B.R. 1988, ApJ, 330, 57

Pierce, M.J. \& Tully, B.R. 1992, ApJ, 387, 47 
Roche, N., Ratnatunga, K., Griffiths, R.E., Im, M., \& Naim, A. 1998, MNRAS, 293, 157.

Schade, D., Lilly, S.J., Crampton, D., Hammer, F., Le Fevre, O., \& Tresse, L. 1995, ApJ, 451, L1.

Schade, D., Lilly, S.J., Le Fevre, O., Hammer, F., \& Crampton, D. 1996, ApJ, 464, 79.

Simard, L., et al. 1999, submitted, astro-ph/9902147.

Somerville, R., Primack, J. 1998, MNRAS, in press, astro-ph/9807277.

Sprayberry, D., Impey, C., Irwin, M.J., \& Bothun, G.D. 1997, ApJ, 481.

Steinmetz, M. \& Bartelmann, M. 1995, MNRAS, 272, 570

Steinmetz, M. and Navarro, J. 1999, ApJ, 513, 550.

Tully, B. \& Fouqué, P. 1985, ApJS, 58, 67.

van den Bosch, F. 1998, ApJ, 507, 601.

Vogt, N.P., Forbes, D.A., Phillips, A.C., Gronwall, C., Faber, S.M., Illingworth, G.D., \& Koo, D.C. 1996, ApJ, 465, L15.

Vogt, N.P., et al. 1997, ApJ, 479, L121.

Wang, B. \& Silk, J. 1994, ApJ, 427, 759.

Warren, M. S., Quinn, P. J., Salmon, J. K. \& Zurek, W. H. 1992, ApJ, 399, 405.

Wyse, R. \& Silk, J. 1989, ApJ, 339, 700.

This preprint was prepared with the AAS IATEX macros v4.0. 

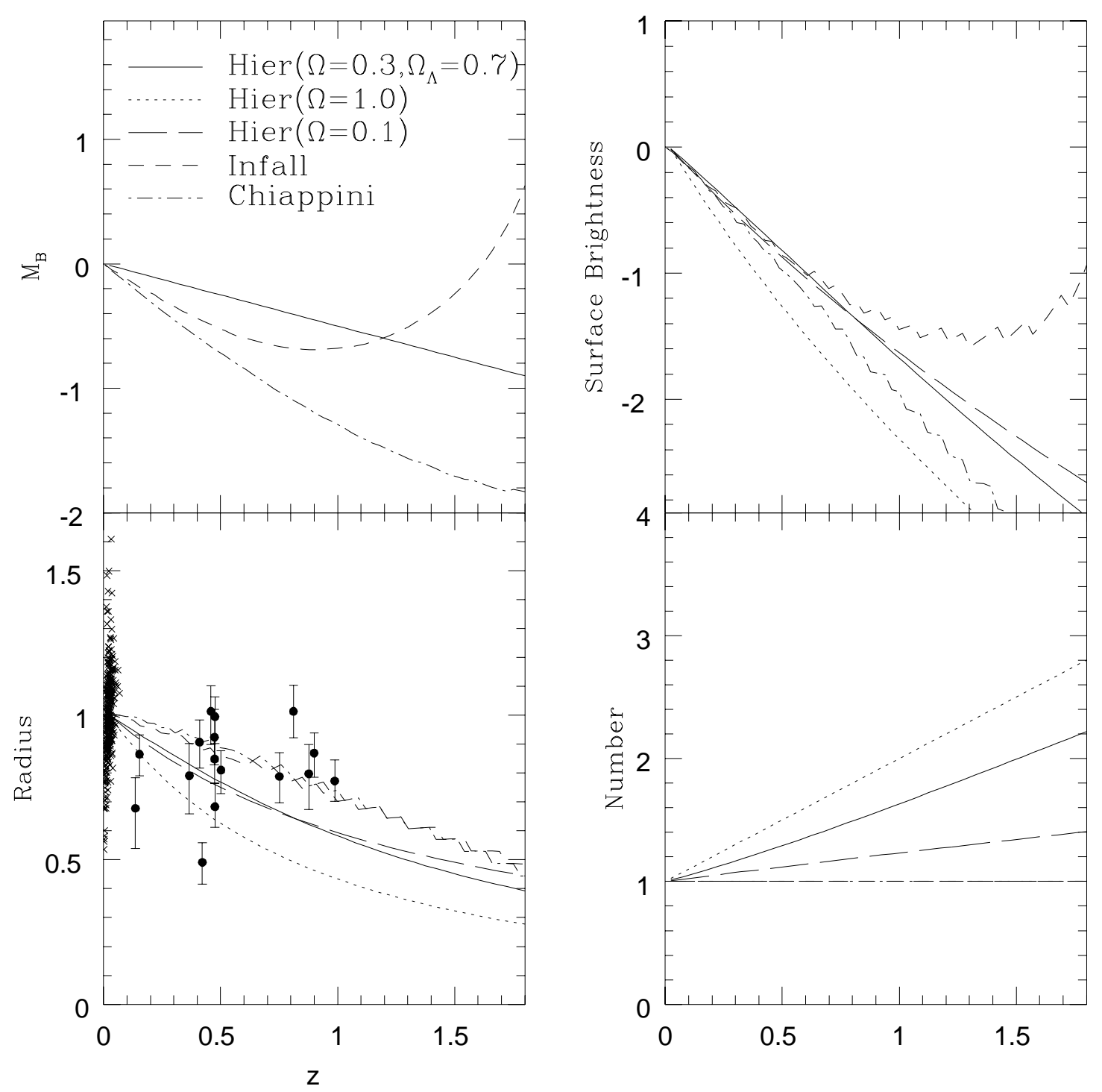

Fig. 1.- Evolution of size, rest-frame $B$ luminosity, number, and surface brightness for a fiducial $L_{*}\left(M \sim 1.2 \cdot 10^{12} M_{\odot}\right)$ galaxy in our $\Omega=0.3 / \Omega_{\Lambda}=0.7$ hierarchical model, our $\Omega=0.1$ hierarchical model (long dashed line), our $\Omega=1$ hierarchical model (dotted line), our infall model (dashed line), and the Chiappini et al. (1997) infall model (dotted-dashed line). Using the $R_{d} / V_{c}$ as a measure of size for a given mass halo, we have added the $z=0$ Courteau (1997) sample and higher-redshift Vogt et al. (1996, 1997) sample to this plot, our scaling the $R_{d} / V_{c}$ values so that the Courteau sample had a fiducial scale length of unity at $z=0$. Both the data and models are presented using $\Omega=0.3, \Omega_{\Lambda}=0.7, H_{0}=70 \mathrm{~km} / \mathrm{s} / \mathrm{Mpc}$, and corrected to unattenuated magnitudes (Tully \& Fouqué 1985). 


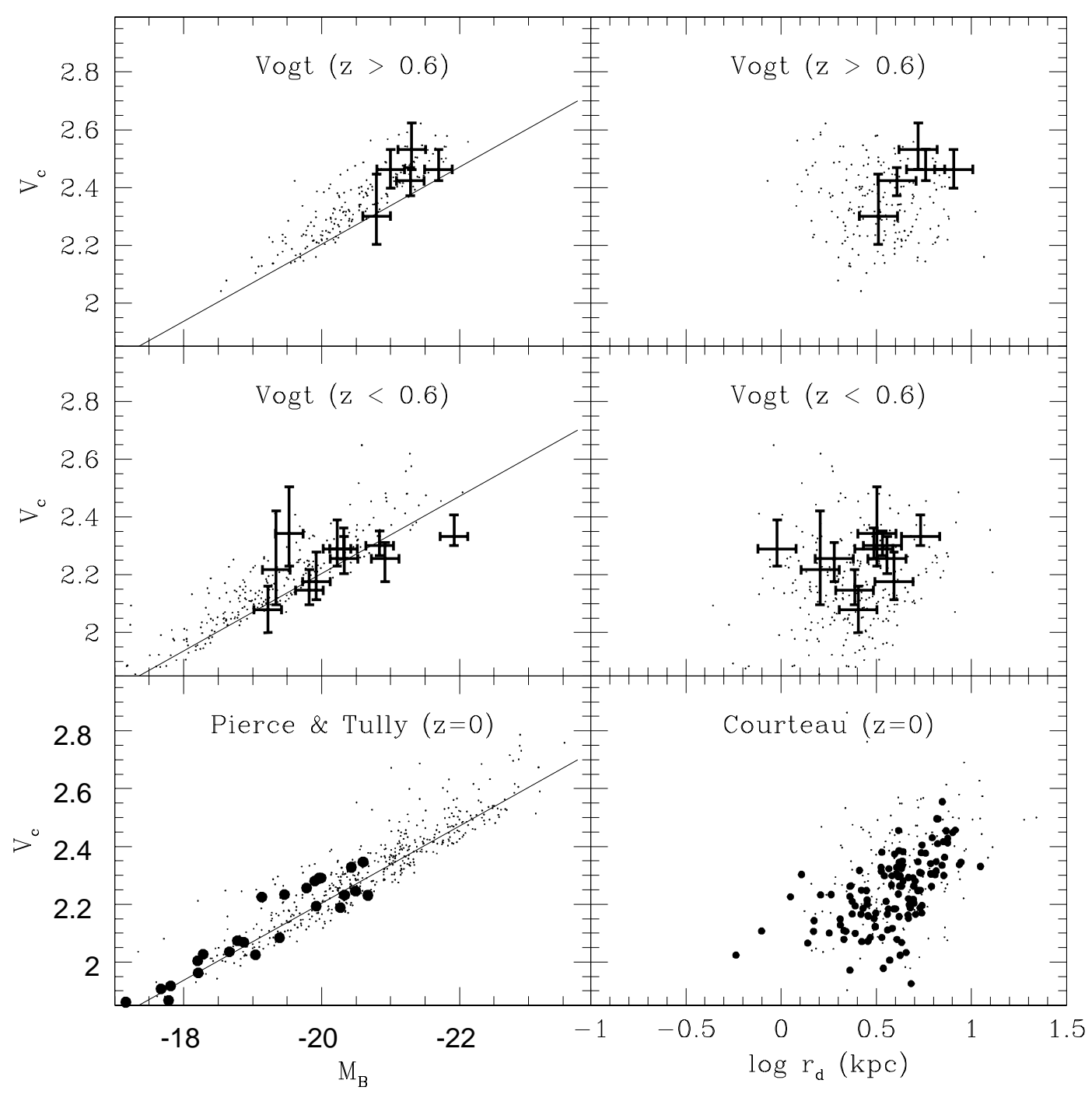

Fig. 2.- Comparison of the observed Tully-Fisher and size-circular velocity relationships as a function of redshifts against the presented $\Omega=0.3, \Omega_{\Lambda}=0.7$ hierarchical model at low and high redshift. The low-redshift Tully-Fisher data is from Pierce \& Tully (1988), the high-redshift data is from Vogt et al. (1996, 1997), the superimposed line is the Pierce \& Tully (1992) Tully-Fisher relationship, and the low-redshift $V_{c}$-size data is from Courteau (1997). 


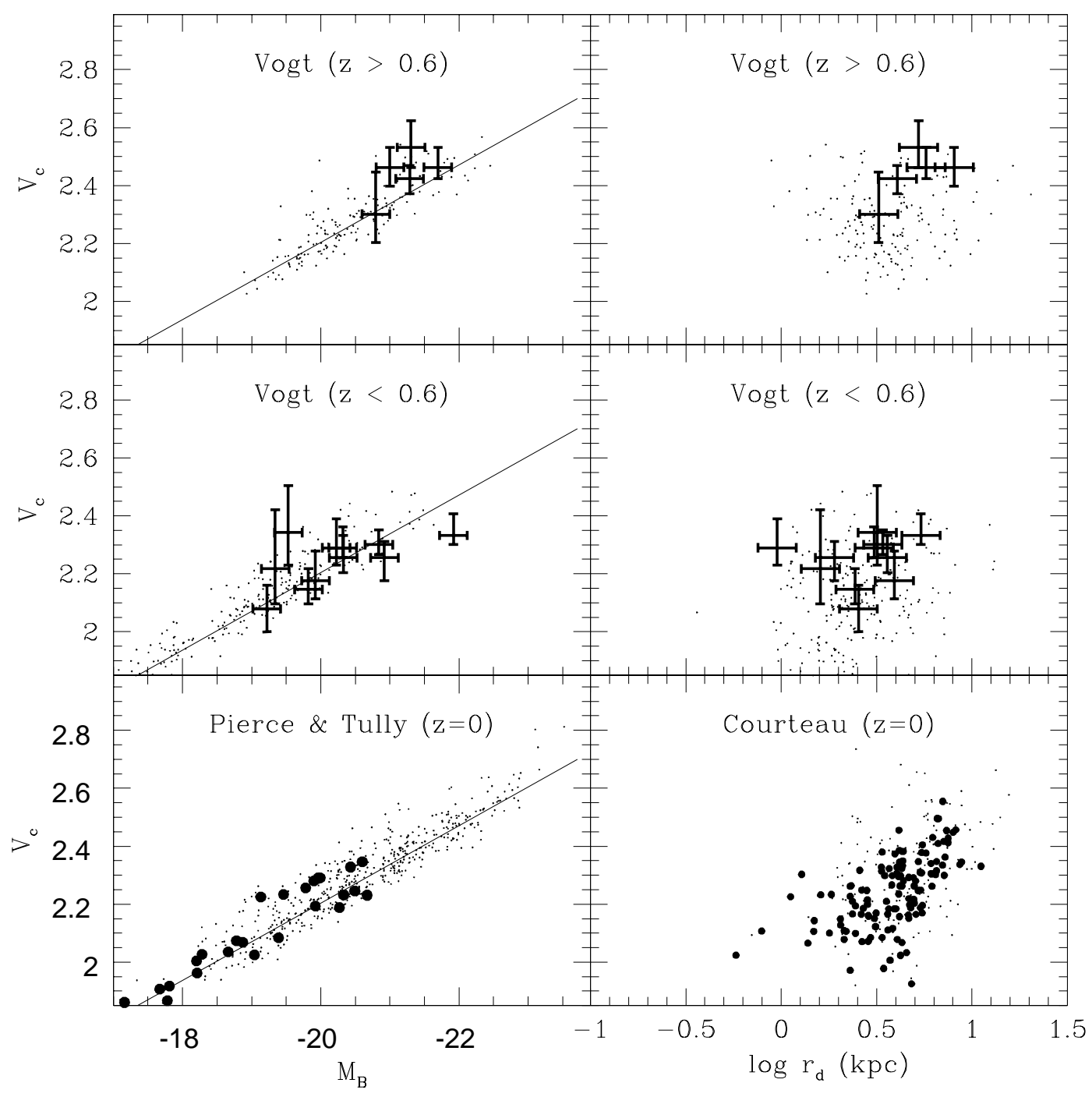

Fig. 3.- Comparison of the observed Tully-Fisher and radius-circular velocity relationships against the presented $\Omega=0.3, \Omega_{\Lambda}=0.7$ infall model at low and high redshift. The data is as in Figure 2. 


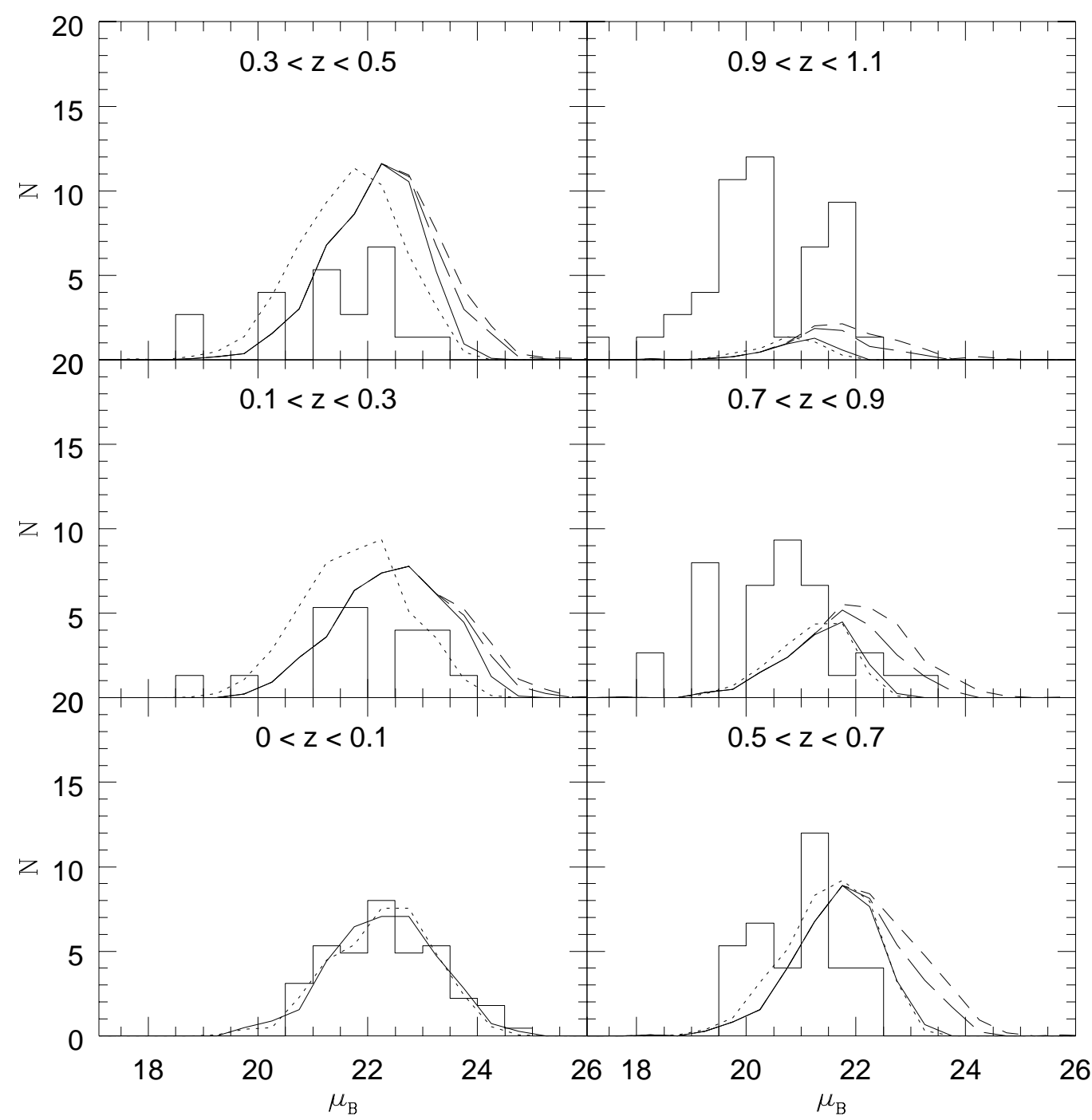

Fig. 4.- Comparison of the observed rest-frame $B$ surface brightness distributions (histogram) with those from our fiducial no-evolution model (solid line), our fiducial noevolution model with constant $M_{b_{J}}=-21$ surface brightness distribution (dotted line), our fiducial no-evolution model without surface brightness selection (dashed line), and our fiducial no-evolution model with the less conservative selection function of Simard et al. (1999) (long dashed line). This illustrates the possible importance of surface brightness selection and an assumed luminosity-surface brightness correlation on the conclusions derived. The local $(z<0.1)$ data is from de Jong \& van der Kruit (1994) and the highredshift $(z>0.1)$ data is from Simard et al. (1999). 


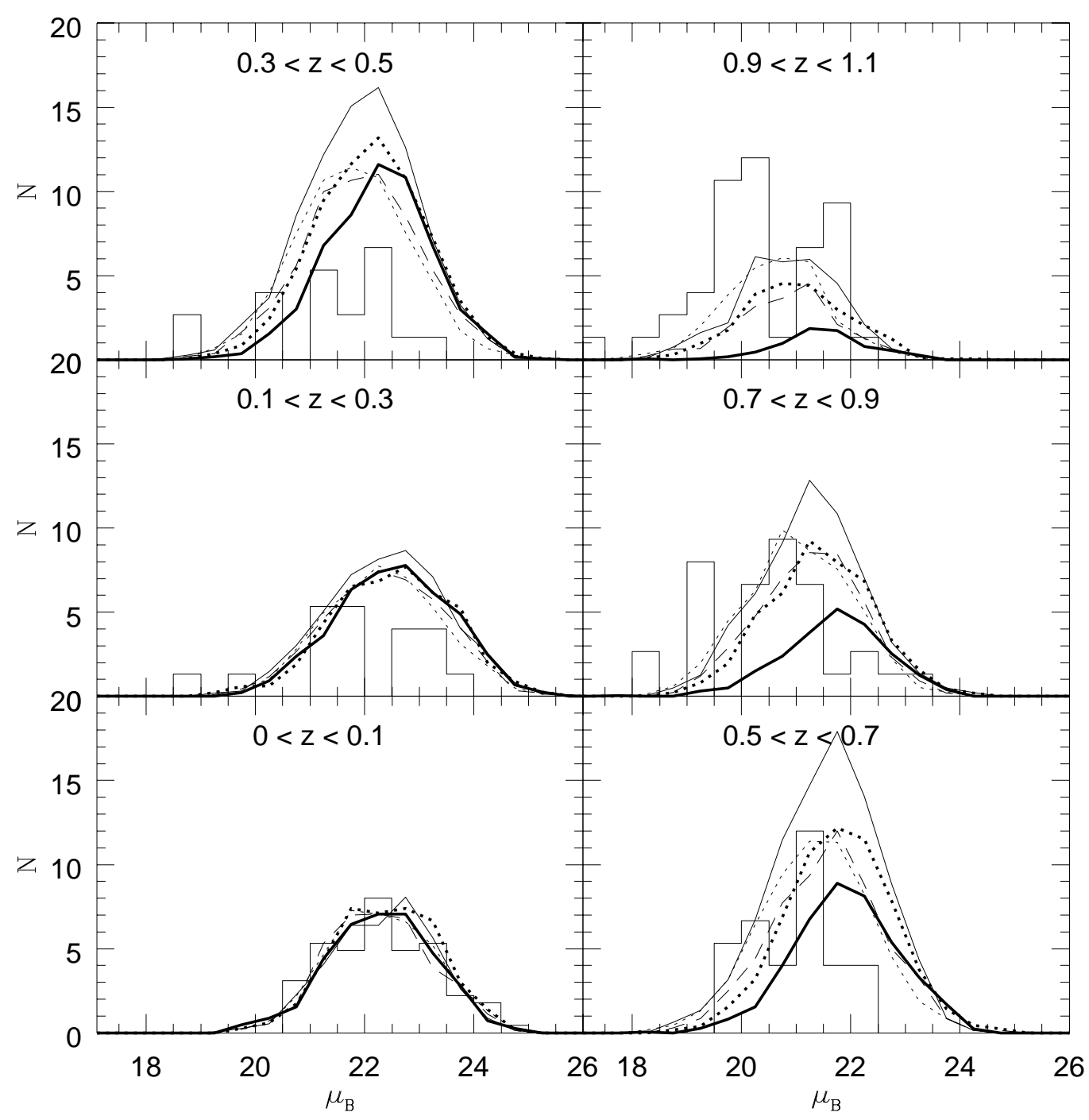

Fig. 5.- Comparison of the observed rest-frame $B$-band surface brightness distributions (histogram) with those from our hierarchical models $\left(\Omega=0.3, \Omega_{\Lambda}=0.7 /\right.$ solid line; $\Omega=0.1 /$ dashed line; $\Omega=1 /$ dotted line), our infall models (thick dotted line), and our fiducial no-evolution model (thick solid line) presented here. The local $(z<0.1)$ data is from de Jong \& van der Kruit (1994) and the high-redshift $(z>0.1)$ data is from Simard et al. (1999). 


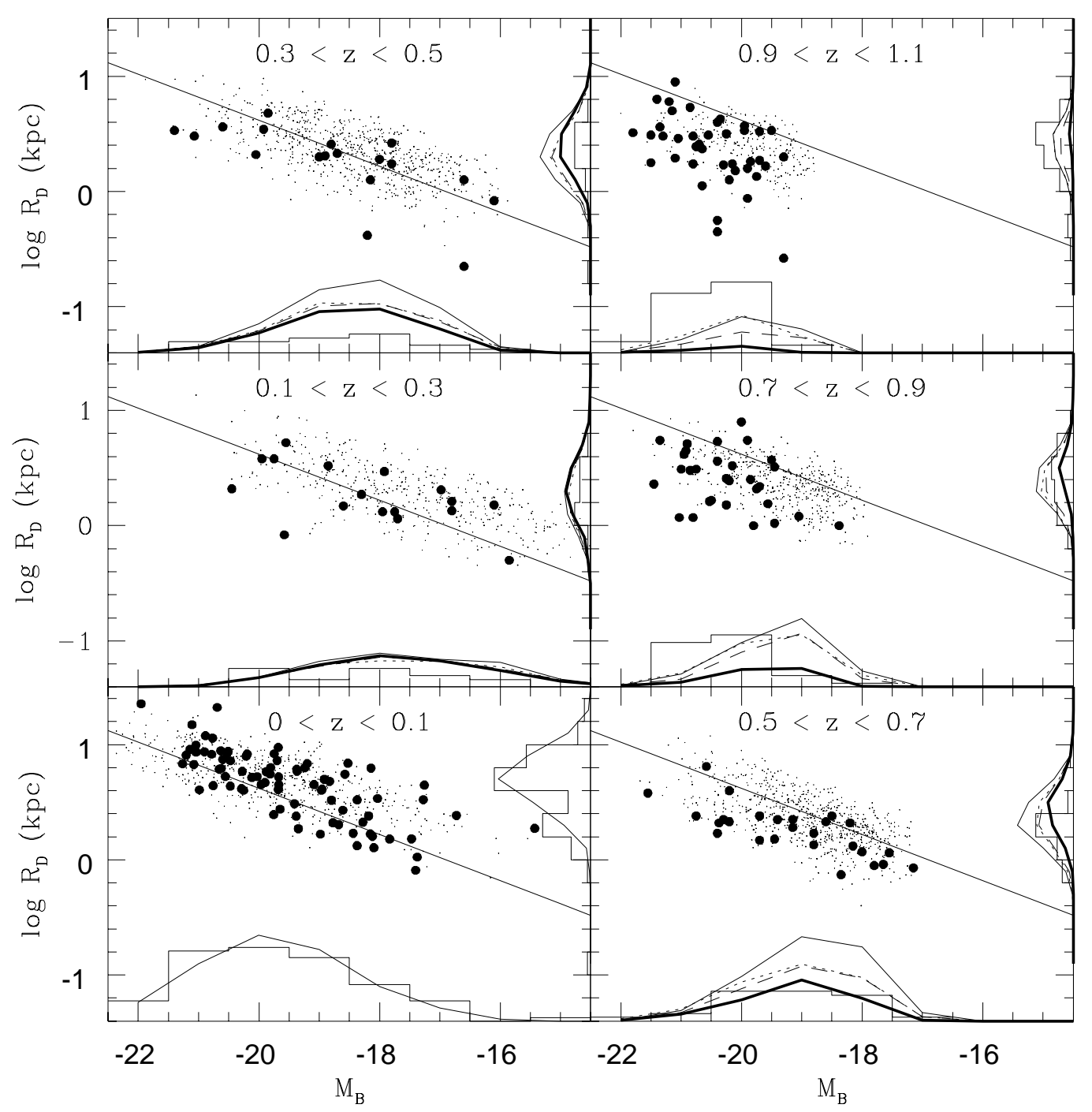

Fig. 6.- Comparison of the observed magnitude-radius (rest-frame $B$ ) distributions with the hierarchical models presented here. The low-redshift data (filled circles) is from de Jong \& van der Kruit (1994) and the high-redshift data (filled circles) is from Simard et al. (1999), and the small dots are the results for the $\Omega=0.3, \Omega_{\Lambda}=0.7$ hierarchical model. Cumulative size and luminosity distributions are presented on the vertical and horizontal axes, respectively, for our $\Omega=0.1$ hierarchical model (dashed line), our $\Omega=1$ hierarchical model (dotted line), our $\Omega=0.3, \Omega_{\Lambda}=0.7$ hierarchical model (solid line), and our fiducial noevolution model (thick solid line) for comparison with the observations (histogram). Both the data and models are presented using $\Omega=0.3, \Omega_{\Lambda}=0.7, H_{0}=70 \mathrm{~km} / \mathrm{s} / \mathrm{Mpc}$, and assuming an inclination of $70 \mathrm{deg}$ (Tully \& Fouqué 1985). 


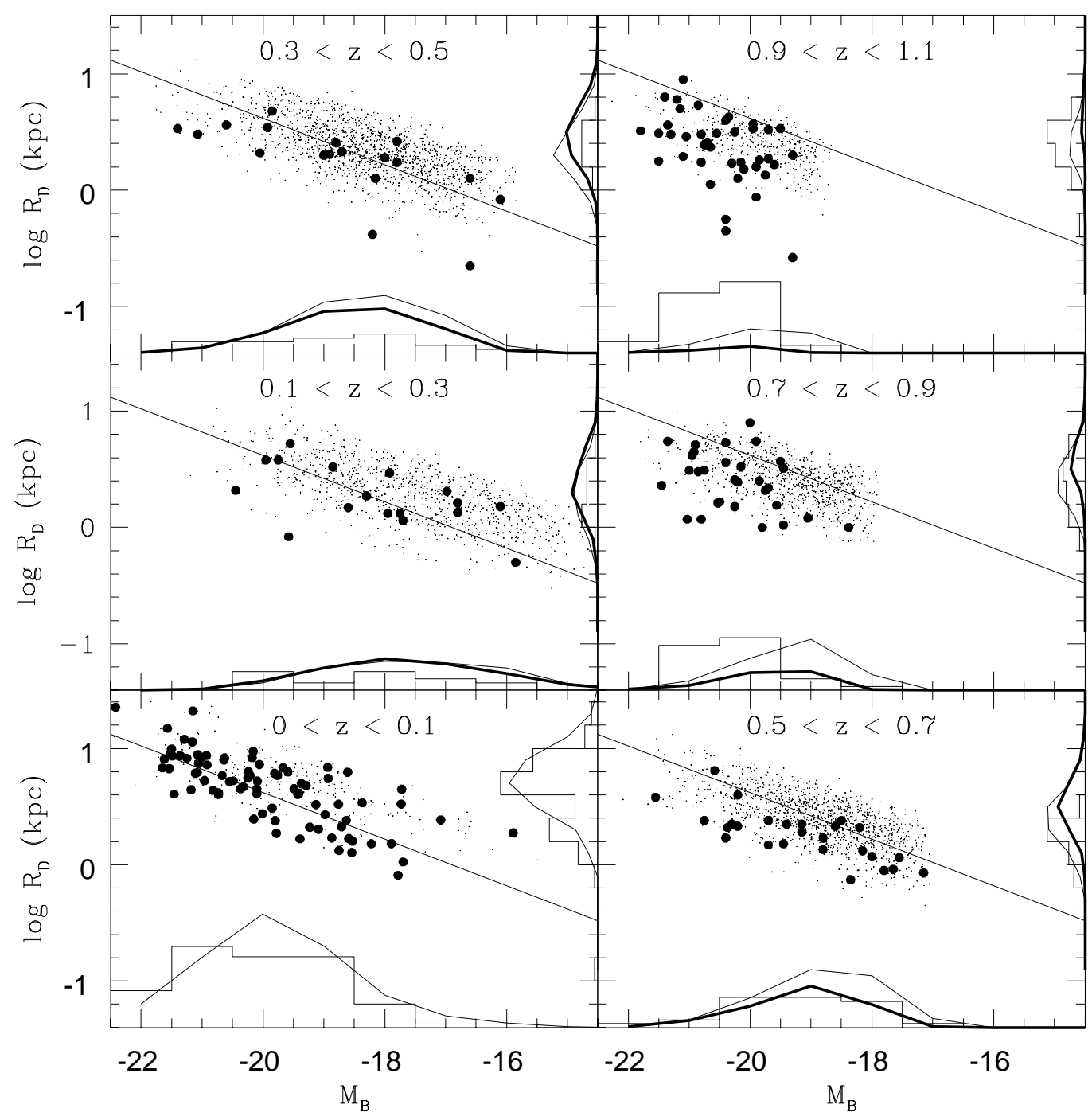

Fig. 7.- Comparison of the observed magnitude-radius (rest-frame $B$ ) distributions with the $\Omega=0.3, \Omega_{\Lambda}=0.7$ infall model presented here $(\S 2.2)$. The data is as on Fig 5 , and the small dots trace out the model distribution. Cumulative size and luminosity distributions are presented for the infall model (solid lines), the no-evolution models (thick line), and the observations (histogram). 


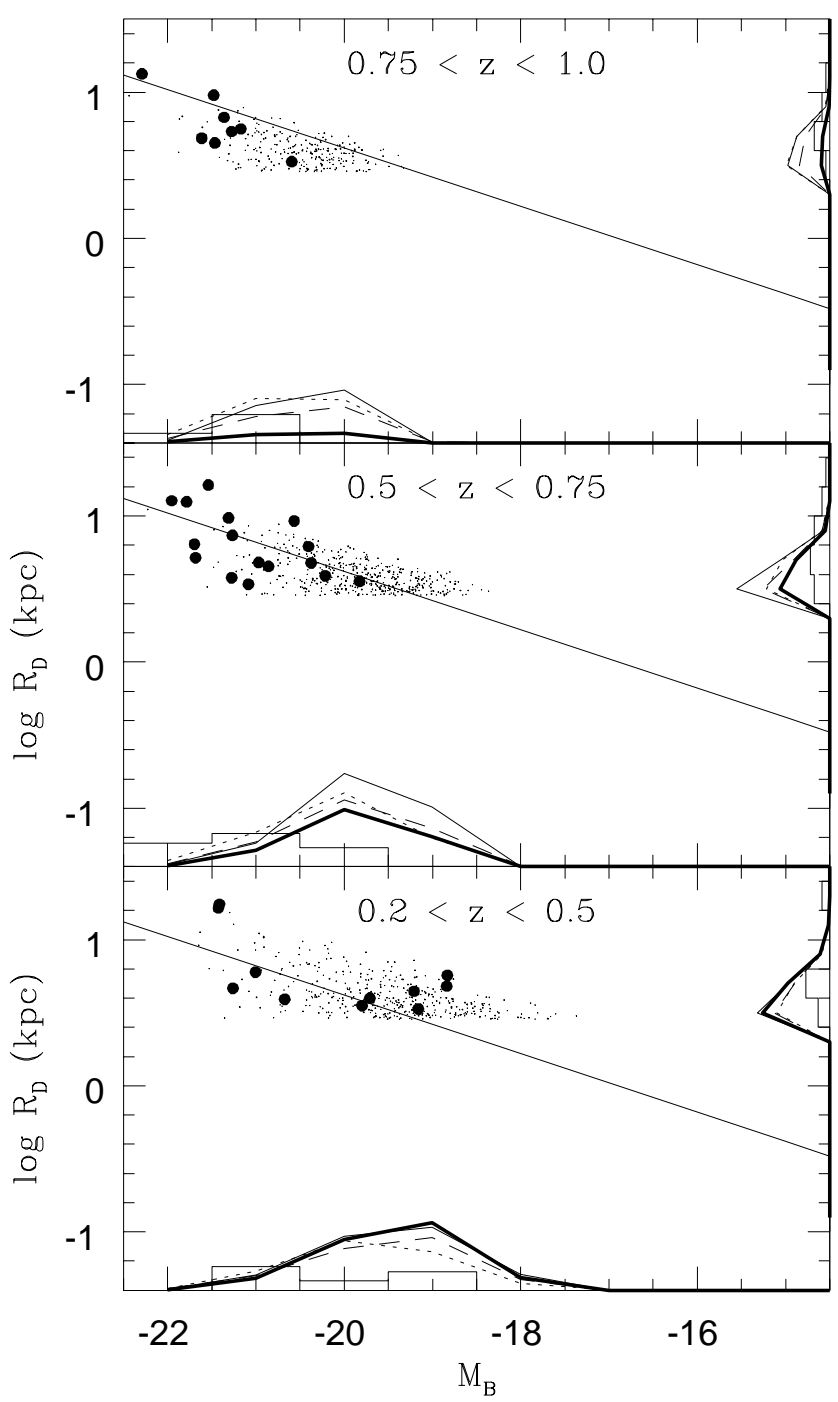

Fig. 8.- Comparison of the observed magnitude-radius (rest-frame $B$ ) distributions from the Lilly et al. (1998) large disk sample with the hierarchical models presented here. The models and data are as in Figure 6. Both the data and models are presented using $\Omega=0.3$, $\Omega_{\Lambda}=0.7, H_{0}=70 \mathrm{~km} / \mathrm{s} / \mathrm{Mpc}$, and assuming an inclination of $70 \mathrm{deg}$ (Tully \& Fouqué 1985). There are an excess of model galaxies at low magnitudes and large sizes relative to the observations. 

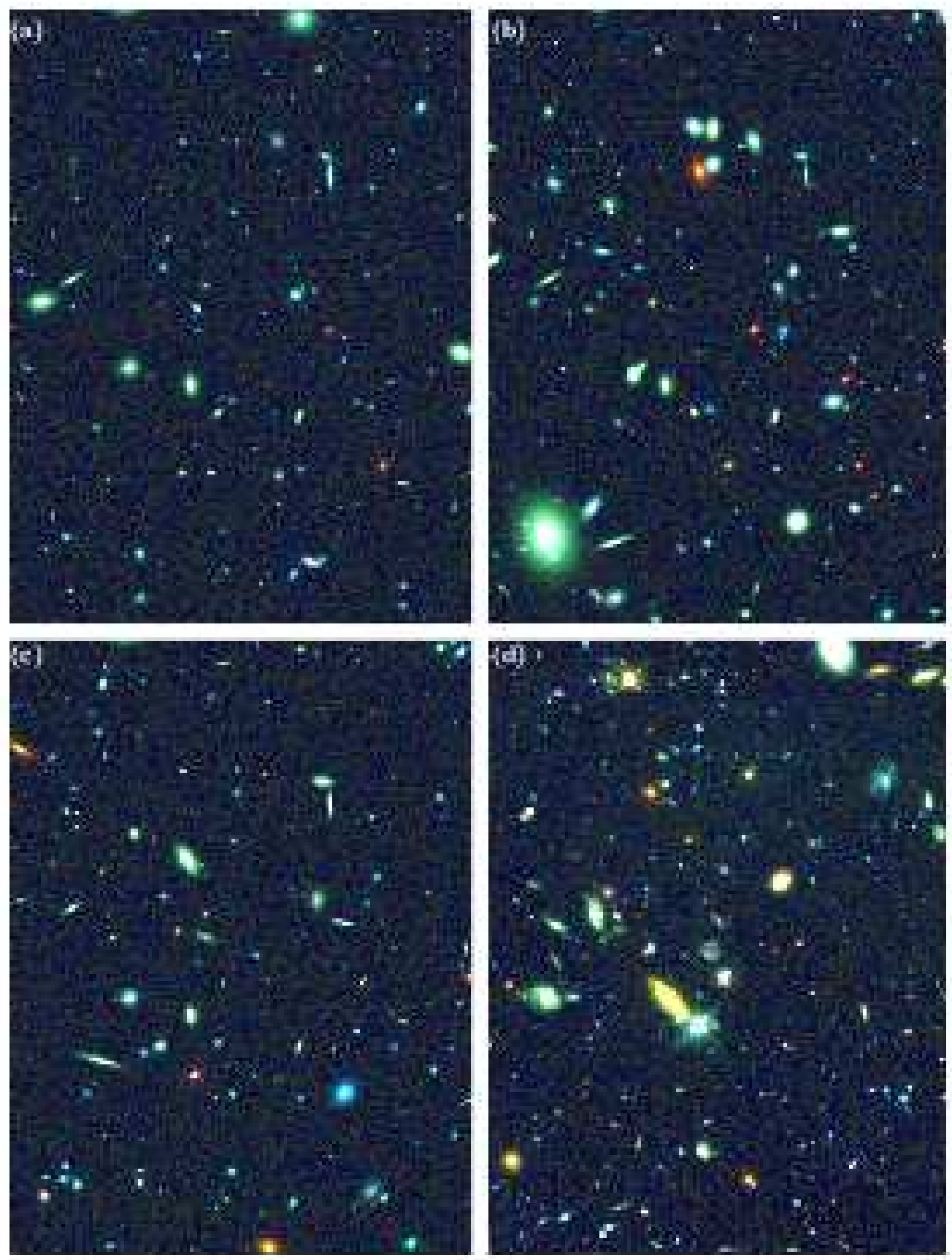

Fig. 9.- Panels (a), (b), (c), and (d) show 60" x 80" color images $\left(I_{F 814 W}, B_{F 450 W}\right.$, and $V_{F 606 W}$ ) for an HDF-depth simulation using our fiducial no-evolution model, an HDFdepth simulation using our $\Omega=1$ hierarchical model, an HDF-depth simulation using our $\Omega=0.3, \Omega_{\Lambda}=0.7$ infall model, and a portion of the actual HDF North and South. Clearly, our no-evolution model has fewer high surface brightness galaxies than the HDF. Our $\Omega=1$ hierarchical model also appears to lack high surface brightness galaxies though the fact that we did not include peculiars and bright ellipticals in our simulations would tend to bias the eye. 